\title{
Laser Alloying Nickel on 4H-Silicon Carbide Substrate to Generate Ohmic Contacts
}

\author{
B. Adelmann ${ }^{*}$, A. Hürner ${ }^{* *}$, T. Schlegel ${ }^{* *}$, A. J. Bauer ${ }^{* * *}$, L. Frey $^{* *}$, R. Hellmann ${ }^{*}$ \\ ${ }^{*}$ University of Applied Sciences Aschaffenburg, Würzburger Str. 45, 64743 Aschaffenburg, Germany \\ E-mail: Benedikt.adelmann@h-ab.de \\ ${ }^{* *}$ Chair of Electron Devices, University of Erlangen-Nuremberg, Germany \\ ${ }^{* * *}$ Fraunhofer Institute for Integrated Systems and Device Technology, \\ Schottkystrasse 10, 91058 Erlangen, Germany
}

\begin{abstract}
To obtain ohmic contacts on silicon carbide semicondanneuctors commonly a thin metal layer is deposited onto the semiconductor wafer, followed by a rapid thermal process to transform the resulting Schottky diode into an ohmic contact. Here, we report on an alternative laser based process replacing the conventional rapid thermal treatment. A continuous wave infrared fiber laser is employed to locally alloy nickel coated silicon carbide to an ohmic contact. Our results show that ohmic contacts are achieved with laser powers between $100 \mathrm{~W}$ and $300 \mathrm{~W}$ over an irradiated area on the order of $7 \mathrm{~mm}$ diameter. Electrical resistances of the generated ohmic contacts are measured down to $6.5 \Omega$, depending on laser power. Temperature dependent measurements of the resistance reveal a negative temperature coefficient of $11 \mathrm{~m} \Omega / \mathrm{K}$.

DOI:10.2961/jlmn.2013.01.0019
\end{abstract}

Keywords: Silicon carbide semiconductor, ohmic contacts, fiber laser, laser surface treatment

\section{Introduction}

Today, most semiconductor devices are produced on silicon substrates with the relevant process steps for silicon based technologies being well established. Among these, also lasers based processes as, e.g., wafer dicing [1] [2], laser alloyingling [3] or via drilling [4] are state of the art. For new high power electronic applications as well as for ultrafast electronic devices, however, silicon technology has physical limitations. In turn, semiconductors based on silicon carbide ( $\mathrm{SiC}$ ) have different advantages over silicon. The most important advantages of silicon carbide are i) the higher band gap of $3.2 \mathrm{eV}$ in $4 \mathrm{H}-\mathrm{SiC}$, ii) the thermal conductivity being twice as high as in silicon, iii) the ten times higher dielectric disruptive strength and iv) the higher saturated electron drift velocity, which is twice as high as in silicon [5] [6]. These properties are beneficial in high power electronics, hot environments and for ultrafast electronics. Unfortunately, however, some important process steps for silicon carbide based devices are not yet well established. For example, the deposition of ohmic contacts is still a topic of current research, being discussed in numerous research articles [7] [8] [9] [10]. These ohmic contacts are most commonly produced by depositing nickel, tungsten, or titanium on the semiconductor using physical vapor deposition (PVD) [7]. However, the electrical contact at the interfacing boundary between the $\mathrm{SiC}$ substrate and the metal behaves as a Schottky diode rather than an ohmic contact. To achieve ohmic behavior, the wafers are usually alloyed in a so called rapid thermal process (RTP) for several minutes at temperatures between $900^{\circ} \mathrm{C}$ and $1000^{\circ} \mathrm{C}$ [11]. During this process, the metal and the semiconductor react to silicides such as $\mathrm{Ni}_{2} \mathrm{Si}$ or $\mathrm{TiSi}_{2}$ depending on the deposited metal and alloying conditions [12]. These alloys are responsible for the ohmic behavior of the contact with n-type ohmic contact resistance being in the range of typi- cally between $10^{-7} \Omega \mathrm{cm}^{2}$ and $10^{-5} \Omega \mathrm{cm}^{2}$, respectively. Ordinarily p-type contacts have a much higher contact resistances. However, this alloying process has the disadvantage to alloy all metallized sections of the wafer on both sides. To circumvent this and to selectively alloy only at specified locations on the wafer, laser irradiation can be used to locally heat the material and initiate the silicidation. A further advantage of laser processing is the low thermal budget which is also compatible with polyimide substrates [13].

During the laser alloying of nickel on a silicon wafer at only $200^{\circ} \mathrm{C}$ the $\mathrm{Ni}_{2} \mathrm{Si}$ phase with a high resistivity $\left(45 \Omega \mathrm{cm}^{2}\right)$ is formed while at $300^{\circ} \mathrm{C}$ the low resistant $\left(7.5 \Omega \mathrm{cm}^{2}\right)$ NiSi phase is generated [3]. Bohac et. al. achieved tungsten silicide $\left(\mathrm{WSi}_{2}\right)$ by treating tungsten on a silicon wafer with a $\mathrm{XeCl}$ excimer laser at $1.8 \frac{\mathrm{J}}{\mathrm{cm}^{2}}$ up to 300 shots [14].

The laser induced generation of ohmic contacts in $6 \mathrm{H}$ $\mathrm{SiC}$ using a pulsed $\mathrm{KrF}$ excimer laser was studied by Nakashima et. al. [15]. With a laser fluence of $2 \frac{\mathrm{J}}{\mathrm{cm}^{2}}$ and 200 shots a contact resistance of $4.3 \times 10^{-5} \Omega \mathrm{cm}^{2}$ was achieved. Similar experiments with a pulsed infrared $\mathrm{Nd}$ :YAG solid state laser were performed by Ota et. al. [16]. In $6 \mathrm{H}-\mathrm{SiC}$ an ohmic contact resistance of $4 \times$ $10^{-5} \Omega \mathrm{cm}^{2}$ with 6 laser pulses at a laser fluence of $30 \frac{\mathrm{J}}{\mathrm{cm}^{2}}$ were achieved.

In this contribution, we report on laser alloying of $4 \mathrm{H}-$ $\mathrm{SiC}$ using a continuous wave (cw) fiber laser. The objective of this study is to achieve ohmic contacts with smallest possible electrical resistance between the semiconductor and the metallization. Opposite to the previously employed pulsed laser irradiation, we additionally aim to avoid any ablation of the thin metal surface layer under continuous 
wave irradiation. Such ablation typically occurs under pulsed laser irradiation as a result of the high energy impinging on the metal within the short pulse duration [17]. In addition, contrary to pulsed laser irradiation with nanosecond pulses and repetition rates ranging from $\mathrm{Hz}$ to $\mathrm{kHz}$, in the cw-regime the material is heated over periods of seconds providing sufficient time for the metal in-diffusion and the formation of silicide. Furthermore, from an industrial application point of view, a continuous fiber laser setup is an attractive alternative to the pulsed excimer laser based approach.

\section{Experimental}

\subsection{Laser System}

In our study, a continuous wave $500 \mathrm{~W}$ single mode fiber laser (IPG Photonics) was used to perform the laser alloying experiments. The emitting wavelength is $1070 \mathrm{~nm}$ in conjunction with a beam propagation factor $\mathrm{M}^{2}$ of $<1.1$. By defocussing the laser beam, its diameter (Gaussian beam profile) is regulated to $7 \mathrm{~mm}$ on top of the surface of the semiconductor. With this beam diameter, several contacts can be processed simultaneously. The experiments are carried out at room temperature with nitrogen as an assist gas that flows onto the semiconductor surface to reduce any possible oxidation.

\subsection{Samples}

We used commercially available 3-inch n-type 4H-SiC wafers (Dow Corning) having a thickness of $300 \mu \mathrm{m}$. For the measurement of the contact resistance, an array of two rectangular nickel contacts with a thickness of $100 \mathrm{~nm}$ was deposited by sputtering onto the wafer. The contacts have an area of $2.56 \mathrm{~mm}^{2}$ for contact A and $1.28 \mathrm{~mm}^{2}$ for contact $\mathrm{B}$, respectively. Individual samples used for the laser treatment and the electrical characterization were laser cut using a $20 \mathrm{~W}$ pulsed fiber laser (IPG Photonics) and have a size of $4 \mathrm{~mm} \times 4 \mathrm{~mm}$.

Figure 1 depicts a simplified cross section of these individual samples with a contact distance of $200 \mu \mathrm{m}$. With a two point current versus voltage measurement (I-V measurement) the contacts resistance is determined for a series of samples that have been alloyed with varying laser powers between $50 \mathrm{~W}$ and $300 \mathrm{~W}$ and with different processing times $(0.5 s-120 s)$, respectively. The contact resistance has been evaluated at different ambient temperatures between $300 \mathrm{~K}$ and $450 \mathrm{~K}$.

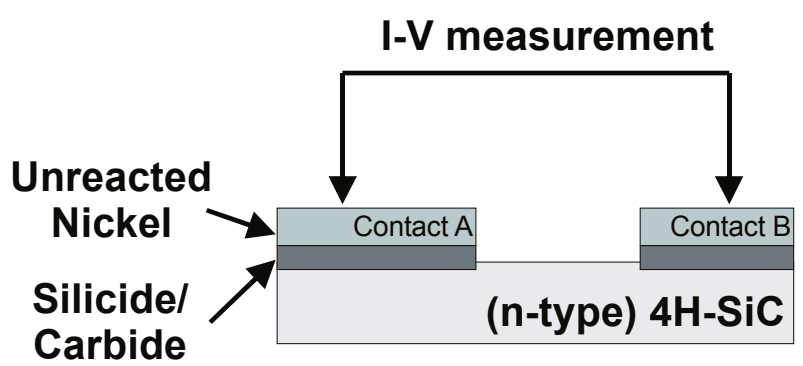

Figure 1 Simplified sample structure of the contacts and I-V measurement in cross section.

\section{Results and discussion}

\subsection{Low power processing}

In our experiments, we do not observe any mechanical fraction at low laser powers up to $100 \mathrm{~W}$, independently of the duration of the laser irradition. We attribute this to the moderate thermal load of the semiconductor material $\mathrm{SiC}$ which is characterized by a high thermal conductivity of up to $400 \frac{W}{m K}$ [5]. For the small sized samples, low power input and high thermal conductivity lead to a small thermal gradient between the irradiated surface and the rear side of the sample as well as between the irradiated center and the edges of the sample. In turn, this low temperature gradient is associated with a small mechanical stress which does not damage the semiconductor sample.

Figure 2 illustrates the I-V measurement between the two contacts of different samples that have been processed with laser powers of $50 \mathrm{~W}$ and $100 \mathrm{~W}$ for 30 seconds and 120 seconds, respectively. At a laser power of $50 \mathrm{~W}$, the I$\mathrm{V}$ characteristic reveals a curved shape indicating a Schottky behavior. With increasing duration of the laser treatment at this power level, the amount of electrical current through the structure increases at constant voltages. However, the curved shaped I-V characteristic typical for the Schottky contact remains. Please note that the sample treated with $50 \mathrm{~W}$ for 30 seconds has almost the same behavior as an untreated sample.

For comparison and to estimate the contact resistance from the measured electrical resistance taken from our experiments, we created a reference sample with a standard rapid thermal process. This reference sample was heated for 2 minutes at $910^{\circ} \mathrm{C}$, resulting in an electrical resistance between the two contacts of $10.3 \Omega$ and a standardized contact resistance of $2.52 \times 10^{-5} \Omega \mathrm{cm}^{2}$. Using these values, we estimate the contact resistance of the laser treated samples by rule of proportion.

At a laser power of $100 \mathrm{~W}$, the I-V diagram shows straight lines that indicate an ohmic contact as a consequence of the silicidation. The contact resistance is determined to be $169 \Omega\left(3.7 \times 10^{-4} \Omega \mathrm{cm}^{2}\right)$ after 30 seconds and $47 \Omega\left(1.0 \times 10^{-4} \Omega \mathrm{cm}^{2}\right)$ after 120 seconds processing time, respectively, i.e. four times longer processing leads to about a quarter of the contact resistance.

In conclusion, these results show that at lower power levels around $50 \mathrm{~W}$ the temperature increase is not sufficient to achieve an ohmic contact independent of the duration of the laser treatment. With increasing laser power, i.e. increasing temperature, the alloying of nickel into the $\mathrm{SiC}$ semiconductor is initiated to an extent that is sufficient to create an ohmic contact. The estimated contact resistance, however, is still higher as compared to a pulsed laser treatment as reported by Nakashima et. al. [15] and Ota et. al. [16]. 


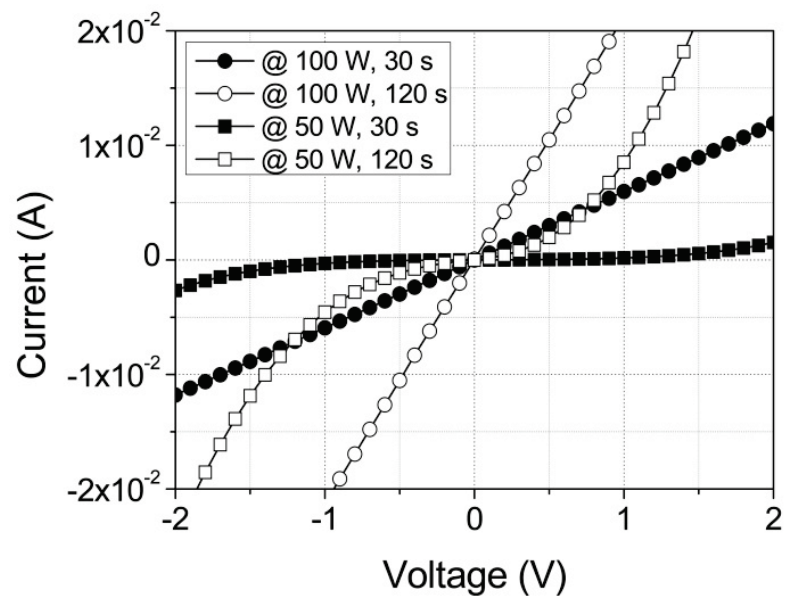

Figure $2 \mathrm{I}-\mathrm{V}$ diagram of the contact resistance treated with different laser powers and varying irradiation time in the low power regime.

\subsection{High power processing}

With increasing laser power the irradiation has to be significantly shortened as the $\mathrm{SiC}$ samples tend to break at higher powers and comparable durations as in the low power regime. Contrary to the low power regime this can be attributed to a higher temperature gradient across the sample that is associated with a high mechanical stress leading to breakage. At higher laser powers the sample surface is heated as quickly as the thermal conductivity is unable to distribute the heat across the entire sample volume. As a result, a significant thermal gradient associated with a mechanical stress is generated leading to breakage. Therefore, at higher laser powers the irradiation time has to be significantly shortened.

Figure 3 show the results of current voltage measurements for a laser treatment in the high power regime with laser powers of $150 \mathrm{~W}$ and $300 \mathrm{~W}$ compared to the measurement at $100 \mathrm{~W}$. In order to avoid a breakage, the irradiation time was shortened to ensure damage free laser treatment. In all cases a linear I-V characteristic, i.e. an ohmic contact due to silicidation, can be found. With higher laser power, an ohmic contact with lower resistance can be obtained even at shorter process times. This can be attributed to a higher diffusion and reaction rate of the nickel caused by the higher temperatures generated in the sample. The lowest ohmic resistance of $6.5 \Omega\left(1.4 \times 10^{-5} \Omega \mathrm{cm}^{2}\right)$ is measured for $300 \mathrm{~W}$ laser power and a processing time of $0.5 \mathrm{~s}$. This is significantly lower than the same sample processed with the RTP method at $910^{\circ} \mathrm{C}$ for 2 minutes with a resulting resistance of $10.3 \Omega\left(2.52 \times 10^{-5} \Omega \mathrm{cm}^{2}\right)$ and lower as compared to a pulsed laser treatment as reported by Nakashima et. al. [15] and Ota et. al. [16].

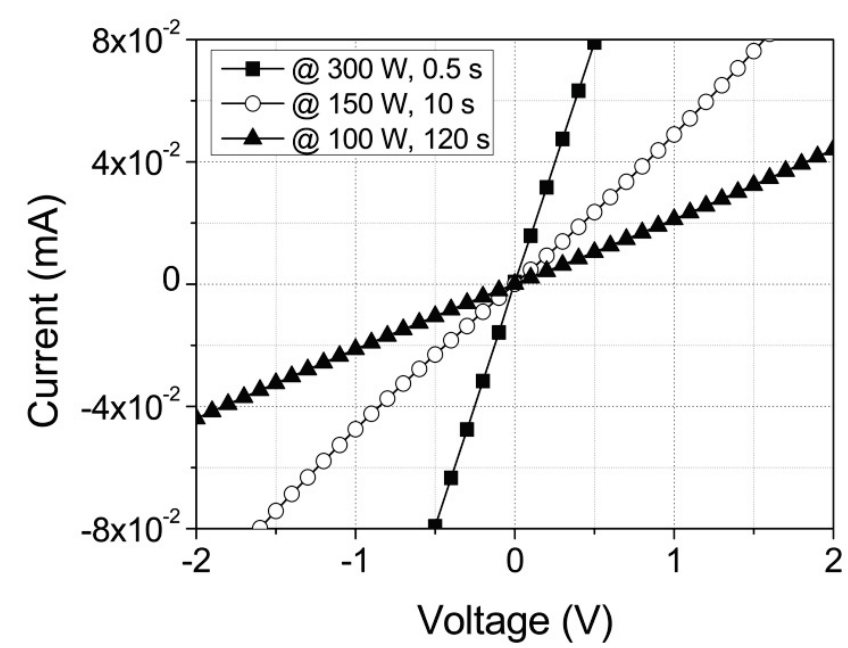

Figure $3 \mathrm{I}-\mathrm{V}$ diagram of the contact resistance treated with high laser powers at different durations.

\subsection{Silicidation depth}

To evaluate the depth of the laser induced silicidation cross section SEM images of the contacts have been examined. Figure 4 shows such a SEM image of a sample that has been laser alloyed with $150 \mathrm{~W}$ for 10 seconds (left) and $300 \mathrm{~W}$ for 0.5 seconds (right).

Figure 4 reveals the layered structure of the irradiated samples. At the top of the SEM image a thick platinum layer was deposited for better contrast (light grey), followed by the $100 \mathrm{~nm}$ thin nickel layer. At the bottom the $\mathrm{SiC}$ substrate can be found. For the sample treated with $300 \mathrm{~W}$ for 0.5 seconds (shown at the right hand side of Figure 4), the alloyed silicide is clearly visible at the interface region between the $\mathrm{SiC}$ substrate and the nickel layer. In this region a structure different to that of both pure silicon carbide and nickel is observed, showing a similar structure to that reported by Alberti et. al. [13], who alloyed silicon with nickel using a pulsed $\mathrm{XeCl}$ excimer laser at a wavelength of $308 \mathrm{~nm}$. In analogy to the findings of Alberti et. al., we assign this structure to silicide, having a thickness of $200 \mathrm{~nm}$. This silicide layer cannot be found in the sample treated with $150 \mathrm{~W}$ for 10 seconds (left hand side of Figure 4).

This interpretation is confirmed by an EDX analysis. For this analysis the Nickel layer on top of the sample shown on the right hand side of Figure 4 was completely removed by an etching step. The EDX analysis of this sample reveals an element concentration of $24 \mathrm{~m} \%$ Nickel and $76 \mathrm{~m} \%$ Silicon, respectively, in turn indicating the silicide formation. Please note that the EDX system employed in this study cannot detect elements with low atomic numbers, as, e.g., Carbon. Therefore, the relatively high concentration of Silicon of $76 \mathrm{~m} \%$ obtained in this measurement can be attributed to the silicon carbide layer. As the penetration depth of the electron beam is higher than the thickness of the formed silicide layer of $200 \mathrm{~nm}$, the silicon carbide substrate contributes to the measured concentrations without detecting a carbon peak. 


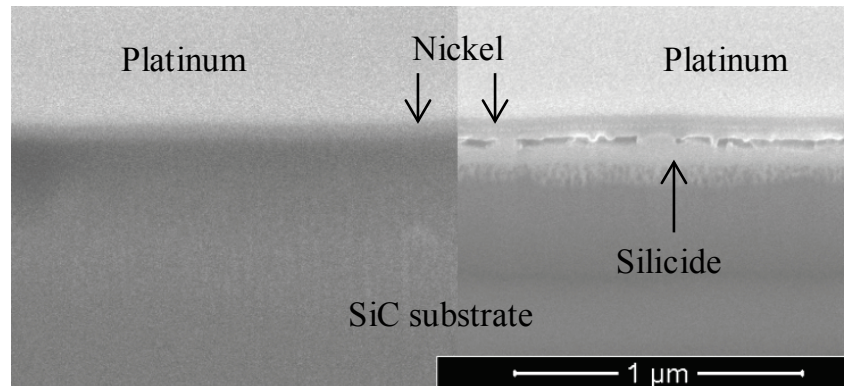

Figure 4 Cross section SEM image of the laser alloyed contact with $150 \mathrm{~W}$ for $10 \mathrm{~s}$ (left) and $300 \mathrm{~W}$ for 0.5 seconds (right).

\subsection{Temperature dependence of the contact resistance}

One of the major advantages of electronics on $\mathrm{SiC}$ substrates is their higher maximum operating temperature. For this reason the contact resistance should be independent from temperature. Figure 5 shows the I-V measurement of the contact resistant of the sample processed with $100 \mathrm{~W}$ for $120 \mathrm{~s}$ at ambient temperatures between $300 \mathrm{~K}$ and $450 \mathrm{~K}$. At all temperatures the contact has a linear, i.e. an ohmic behavior. The contact resistance decreases slightly with increasing temperature with a temperature coefficient of $11 \mathrm{~m} \Omega / \mathrm{K}$. This behavior might be attributed to a higher carrier mobility in the semiconductor at higher temperatures in this temperature regime [6]. However, further studies are needed to analyze this temperature dependence.

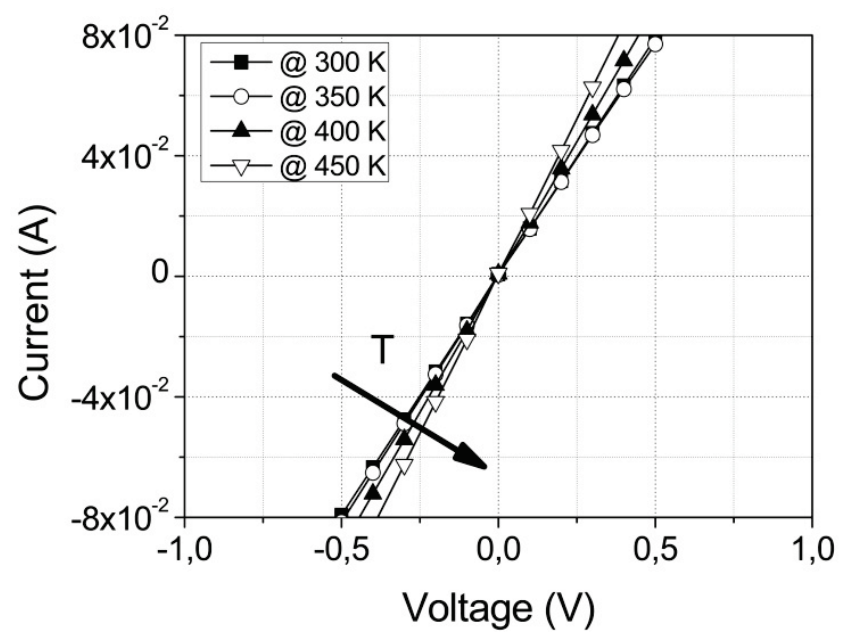

Figure $5 \mathrm{I}-\mathrm{V}$ measurement for different temperatures for the sample treated with $100 \mathrm{~W}$ for $120 \mathrm{~s}$.

\section{Conclusion}

We report on laser alloying of ohmic contacts in $4 \mathrm{H}-$ $\mathrm{SiC}$ semiconductors by using a continuous wave fiber laser. We find that the laser induced silicidation of nickel and silicon carbide under continuous wave irradiation can be separated into two different regimes. At laser powers below $150 \mathrm{~W}$, the transformation from a Schottky diode to an ohmic contact depends strongly on the irradiation time with ohmic contacts being possible only after $120 \mathrm{~s}$. With increasing laser power the necessary irradiation time drops significantly to $0.5 \mathrm{~s}$ at $300 \mathrm{~W}$. The lowest contact resistance of $6.5 \Omega$ is measured at $300 \mathrm{~W}$ laser power and $0.5 \mathrm{~s}$ processing time. The alloyed contacts show an ohmic behavior even at higher temperatures up to $450 \mathrm{~K}$ with a temperature coefficient of $-11 \mathrm{~m} \Omega / \mathrm{K}$.

\section{References}

[1] B. Richerzhagen, R. Housh and F. Wagner, "Water Jet Guided Laser Cutting: a Powerful Hybrid Technology for Fine Cutting and Grooving," ALACAdvanced Laser Applications Conference \& Exposition, 2004.

[2] J. Manley, R. Housh and F. Wagner, "water- guided lasers create clean cuts," Laser Focus World, pp. 1518, 52004.

[3] W. S. Yoo, T. Fukada and I. J. Malik, "Changes in Optical Properities during Nickel Silicide Formation and Potential Impact on Process Results using Various Heating Methods," Advanced Thermal Processing of Semiconductors, 14th IEEE International Conference on, pp. 243 - 249, 10102006.

[4] M. Farsari, G. Filippidis, S. Zoppel, G. Reider and C. Fotakis, "Efficient Femtosecond lasesr micromachining of bulk 3C-SiC," Journal of Micromechanics and Microengineering, pp. 17861789, 152005.

[5] M. Ostling, "High power devices in wide bandgap semiconductors," SCIENCE CHINA, p. 1087-1093, 5 2011.

[6] T. Mnatsakanov, L. Pomortseva and S. Yurkov, "Semiempirical model of carrier mobility in silicon carbide for analyzing its dependence on temperature and doping level," Physics and Astronomy, pp. 394397, 4352001.

[7] W. Daves, A. Krauss, V. Häublein, A. Bauer and L. Frey, "Enhancement of the Stability of Ti and Ni Ohmic Contacts to $4 \mathrm{H}-\mathrm{SiC}$ with a Stable Protective Coating for Harsh Environment Applications," Journal of ELECTRONIC MATERIALS, pp. 19901997, 9402011.

[8] C. Hallin, R. Yakimova, B. Pecz, A. Georgieva and T. Marinova, "Improved Ni Ohmic Contact on n-Type 4H-SiC," Journal of Electronic Materials, pp. 119122, 3261997.

[9] A. Virshup , F. Liu, D. Lucko, K. Buchholt, A. Spetz and L. Porter, "Improved Thermal Stability Observed in Ni-Based Ohmic Contacts to n-Type SiC for HighTemperature Applications," Journal of ELECTRONIC MATERIALS, pp. 400-405, 4402011.

[10] S. Duman, S. Dogan, B. Gürbulak and A. Türüt, "The barrier-height inhomogeneity in identically prepared Ni/n-type 6H-SiC Schottky diodes," Applied Physics, p. 337-340, 912008.

[11] S. Tanimoto, H. Okushi and K. Arai, "Ohmic Contacs for Power Devices on SiC," in Silicon Carbide, Springer, 2004, pp. 652-658.

[12] S. Ferrero, Albonico A., U. Meotto, G. Rombola, S. Porro, F. Giorgis, D. Perrone, L. Scaltrito, E. Bontempi, L. Depero, G. Richeiri and L. Merlin, "Phase Formation at Rapid Thermal Annealing of Nickel Contacts on C-face n-type 4H-SiC," Materials Science Forum, pp. 483-485, 7332005.

[13] A. Alberti, A. La Magna, M. Cuscuna, G. Fortunato , 
C. Spinella and V. Privitera, "nickel-affectec silicon crystallization and silicidation on polyimide by multipulse excimer laser annealing," Journal of Applied Physics, pp. 1235111-1235116, 121082010.

[14] V. Bohac, E. Anna, G. Leggieri, S. Luby, A. Luches, E. Majakova and M. Martino, "Tungsten Silicide Formation by $\mathrm{XeCl}$ Excimer Laser Irradiation of W/Si Samples," Aplied Physics A, pp. 391-396, 561993.

[15] K. Nakashima, O. Eryu , S. Ukai, K. Yoshida and M. Watanabe, "Improved Ohmic Contacts to $6 \mathrm{H}-\mathrm{SiC}$ by Pulsed Laser Processing," Materials Science Forum, pp. 1005-1008, 338-432 2000.

[16] Y. Ota, Y. Ikeda and M. Kitabatake, "Laser Alloying for Ohmic Contacs on $\mathrm{SiC}$ at Roop Temperature," Materials Science Forum, pp. 783-786, 264-268 1998.

[17] D. Dhupal, B. Doloi and B. Bhattacharyya, "Parametric analysis and optimization of $\mathrm{Nd}: \mathrm{YAG}$ laser micro-grooving of aluminum titanate $\mathrm{Al} 2 \mathrm{TiO} 5$ ceramics," International Journal of Advanced Manufacturing Technology, p. 883-893, 2012.

(Received: November 21, 2012, Accepted: January 26, 2013) 